\title{
A Controller Architecture With Anti-Windup
}

\section{Niemann, Henrik}

\section{Published in:}

IEEE Control Systems Letters

Link to article, DOI:

10.1109/LCSYS.2019.2922712

Publication date:

2020

Document Version

Peer reviewed version

Link back to DTU Orbit

Citation (APA):

Niemann, H. (2020). A Controller Architecture With Anti-Windup. IEEE Control Systems Letters, 4(1), $139-144$. [8736334]. https://doi.org/10.1109/LCSYS.2019.2922712

\section{General rights}

Copyright and moral rights for the publications made accessible in the public portal are retained by the authors and/or other copyright owners and it is a condition of accessing publications that users recognise and abide by the legal requirements associated with these rights.

- Users may download and print one copy of any publication from the public portal for the purpose of private study or research.

- You may not further distribute the material or use it for any profit-making activity or commercial gain

- You may freely distribute the URL identifying the publication in the public portal

If you believe that this document breaches copyright please contact us providing details, and we will remove access to the work immediately and investigate your claim 


\title{
A controller architecture with anti-windup
}

\author{
Henrik Niemann
}

\begin{abstract}
This paper presents a framework for anti-windup controllers based on the Youla-Jabr-Bongiorno-Kucera (YJBK) parameterization. Applying this architecture gives an additional YJBK matrix transfer function related to the input saturation. This additional YJBK transfer function can be applied for optimizing the feedback loop around the input saturation. Further, the connection with other anti-windup controller architectures is also considered in this paper.
\end{abstract}

Index Terms-Control system architecture, linear systems

\section{INTRODUCTION}

$\mathbf{S}$ ATURATION in control systems is a known problem. Almost every system includes input saturation due to the limitation in the actuators. It is therefore relevant to consider a system with input saturation. This has been investigated in a large number of papers and books in connection with traditional control, as well as in connection with optimal and robust control. Throughout the years, a number of results dealing with analysis and design of anti-windup controllers for actuator saturates have been published. These methods include different design methods [1], [3], [4], [5], [8], [13], [18], [19], different controller architectures, as well as the robustness aspect in anti-windup controller design, [2], [7], [17], to mention some of the central papers in the area. Some open problems are discussed in the tutorial papers [15], [20].

The main focus in this paper is to apply the YJBK controller architecture in connection with input saturation. The YJBK architecture has previously been applied in connection with saturation, see e.g. [14] and [7]. In [14], the YJBK parameterization is included in an existing anti-windup controller, and applied for the optimization of the anti-windup part. Equivalent in [7], the YJBK parameterization is included in an existing anti-windup controller with respect to robustness in the closed-loop system.

In this paper, the YJBK architecture is applied directly in connection with input saturation. This can be done by using some known results from the YJBK parameterization, [9], [10], [16]. Together with the implementation of the YJBK controller described in [11], a YJBK architecture including an anti-windup part, gets a simple structure.

The relation with other anti-windup architectures is also considered. The anti-windup architecture by Weston and Postlethwaite (named as the W-P architecture in the following) described in e.g. [4], [7], [13], [19] and the architecture by Kothare et.al. described in [6] is considered. It is shown that the W-P architecture and the YJBK architecture are equivalent. A W-P controller can be implemented in the YJBK architecture as well as the other way around. Further, it is

H. Niemann is with Dept. of Electrical Engineering, Automation and Control, Build. 326, Technical University of Denmark, DK-2800 Kgs. Lyngby, Denmark, hhn@elektro.dtu.dk shown that the architecture by Kothare et.al is as a special case of the W-P architecture and the YJBK architecture.

The rest of this paper is organized as follows. The system set-up and some preliminary results are given in Section II. The saturation setup described in the YJBK architecture is given in Section III. The relation between different antiwindup controllers is described in Section IV. A closed-loop stability is analyzed in Section V. An example is given in Section VI, and the paper is closed with a conclusion in Section VII.

\section{System Setup and Preliminary Results}

Let a general system be given by:

$$
\Sigma:\left\{\left(\begin{array}{l}
z \\
y
\end{array}\right)=\left(\begin{array}{ll}
G_{z w} & G_{z u} \\
G_{y w} & G_{y u}
\end{array}\right)\left(\begin{array}{l}
w \\
u
\end{array}\right)\right.
$$

where $w \in \mathcal{R}^{k}$ is an external input vector, $u \in \mathcal{R}^{m}$ is the control input signal vector, $z \in \mathcal{R}^{k}$ is an external output vector, and $y \in \mathcal{R}^{p}$ is the measurement vector.

A state space description of (1) is given by:

$$
\Sigma:\left\{\left(\begin{array}{c}
\dot{x} \\
z \\
y
\end{array}\right)=\left(\begin{array}{c|cc}
A & B_{w} & B_{u} \\
\hline C_{z} & D_{z w} & D_{z u} \\
C_{y} & D_{y w} & D_{y u}
\end{array}\right)\left(\begin{array}{c}
x \\
w \\
u
\end{array}\right)\right.
$$

where $x \in \mathcal{R}^{n}$ is the state vector

The external output $z$ and the external input $w$ be connected through the matrix $\Delta$, i.e.

$$
w=\Delta z
$$

$\Delta$ represent uncertainties in the system. The general system $\Sigma_{\Delta}$ including uncertainties is given by:

$$
\Sigma_{\Delta}=\mathcal{F}_{u}(\Sigma, \Delta)
$$

where $\mathcal{F}_{u}(\cdot, \cdot)$ is an upper linear fractional transformation (LFT), [12].

The system is controlled by a stabilizing feedback controller given by:

$$
\Sigma_{C}:\{u=K y
$$

The coprime factorization of the nominal system $G_{y u}$ from (1), and the stabilizing controller $K$ from (5) are given by:

$$
\begin{array}{llc}
G_{y u} & =N M^{-1}=\tilde{M}^{-1} \tilde{N}, & N, M, \tilde{N}, \tilde{M} \in \mathcal{R} \mathcal{H}_{\infty} \\
K & =U V^{-1}=\tilde{V}^{-1} \tilde{U}, & U, V, \tilde{U}, \tilde{V} \in \mathcal{R} \mathcal{H}_{\infty}
\end{array}
$$

where the eight matrices in (6) must satisfy the double Bezout equation given by, see [16]:

$$
I=\tilde{Z} Z=Z \tilde{Z}
$$


where the two Bezout matrices are given by

$$
Z=\tilde{Z}^{-1}=\left(\begin{array}{cc}
M & U \\
N & V
\end{array}\right), \tilde{Z}=Z^{-1}=\left(\begin{array}{cc}
\tilde{V} & -\tilde{U} \\
-\tilde{N} & \tilde{M}
\end{array}\right)
$$

Based on the above coprime factorization, a parameterization of all controllers that stabilize the system in terms of a stable YJBK matrix transfer function $Q$. Using a right factored form, all stabilizing controllers are given by [16]:

$$
K(Q)=(U+M Q)(V+N Q)^{-1}, Q \in \mathcal{R H}_{\infty}
$$

or by using a left factored form:

$$
K(Q)=(\tilde{V}+Q \tilde{N})^{-1}(\tilde{U}+Q \tilde{M}), Q \in \mathcal{R H}_{\infty}
$$

Using the Bezout equation, the controller given either by (8) or by (9) can be realized as a linear fractional transformation in the parameter $Q$ :

$$
K(Q)=\mathcal{F}_{l}\left(\left(\begin{array}{cc}
U V^{-1} & \tilde{V}^{-1} \\
V^{-1} & -V^{-1} N
\end{array}\right), Q\right)=\mathcal{F}_{l}\left(J_{K}, Q\right)
$$

The YJBK parameterization for the left factored form in (9) is shown in Fig. 1, where $\tilde{\mathcal{Z}}$ and $\mathcal{Q}$ are given by:

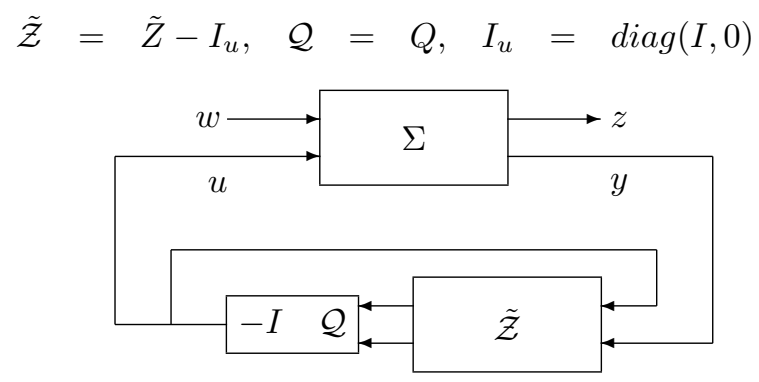

Fig. 1. An implementation of the YJBK parameterization based on the left factored form where $\tilde{\mathcal{Z}}$ and $\mathcal{Q}$ are given by (11).

Fig. 1 gives a direct relation between the Bezout equation and the YJBK controller architecture. This representation has been discussed in more details in [11]. The output vectors from $\tilde{\mathcal{Z}}$ is given by

$$
\left(\begin{array}{l}
-u \\
\varepsilon_{\mathcal{Q}}
\end{array}\right)=\tilde{\mathcal{Z}}\left(\begin{array}{l}
u \\
y
\end{array}\right)
$$

where $\varepsilon_{Q}$ is the standard residual vector.

Further, we need some results from [10] dealing with coprime factorization of systems including additional sensors. Let additional sensors be included in the original system $\Sigma$ in (1) resulting in an extra set of measurements given by $y_{s} . \Sigma$ is then given by:

$$
\Sigma_{e x t}:\left\{\left(\begin{array}{c}
z \\
\hline y \\
y_{s}
\end{array}\right)=\left(\begin{array}{c|c}
G_{z w} & G_{z u} \\
\hline G_{y u} & G_{y u} \\
G_{y_{s} w} & G_{y_{s} u}
\end{array}\right)\left(\begin{array}{c}
w \\
u
\end{array}\right)\right.
$$

where $y_{s} \in \mathcal{R}^{p_{s}}$. The coprime factorization of the extended system is then given by the following Bezout matrices, [10]:

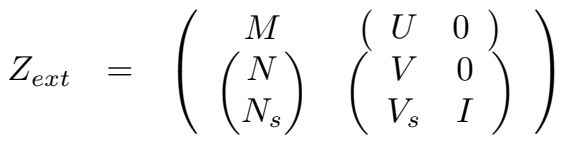

$$
\begin{aligned}
& \tilde{Z}_{e x t}=\left(\begin{array}{cc}
\tilde{V} & \left(\begin{array}{cc}
-\tilde{U} & 0 \\
\tilde{N} \\
-\left(\begin{array}{c}
\tilde{M} \\
\tilde{N}_{s}
\end{array}\right) & 0 \\
\tilde{M}_{s} & I
\end{array}\right)
\end{array}\right)
\end{aligned}
$$

Further, the two matrix transfer functions $\tilde{\mathcal{Z}}$ and $\mathcal{Q}$ in (11) are given by:

$$
\tilde{\mathcal{Z}}=\tilde{Z}_{e x t}-I_{u} \quad \mathcal{Q}=\left(\begin{array}{ll}
Q & Q_{s}
\end{array}\right)
$$

where $Q_{s}$ is related to the the extra measurements including in the system.

It is also possible to derive a parameterization of all systems that are stabilized by one controller in terms of a stable matrix transfer function $S$, i.e. the dual YJBK parameterization. The dual YJBK parameterization is given by [9], [16]:

$$
G_{y u}(S)=(\tilde{M}+S \tilde{U})^{-1}(\tilde{N}+S \tilde{V}), S \in \mathcal{R H}_{\infty}
$$

using the left form. There exists also a right form for the dual YJBK parameterization. Further, $S$ is given as an upper LFT by, [16]:

$$
S=\mathcal{F}_{u}\left(J_{K}, G_{y u}(S)\right)
$$

The matrix transfer function $S$ is a function of the uncertainties in the system described with $\Delta$ as described in (4). $S(\Delta)$ is given by, [9]:

$$
S(\Delta)=\tilde{M} G_{y w} \Delta\left(I-\left(G_{z w}+G_{z u} U \tilde{M} G_{y w}\right) \Delta\right)^{-1} G_{z u} M
$$

Note that the dual YJBK matrix transfer function $S$ can be applied in different connections. From [9], [16], the closedloop uncertain system is stable if the nominal closed-loop system is stable and the resulting $S$ is stable. Here in the following, $S$ will be used in connection with analysis of closed-loop stability when the YJBK controllers includes an anti-windup part.

\section{SATURATION SET-UP}

Consider now the system in (1) with a saturated control input vector given by $u_{s}$. The saturated input vector $u_{s}$ is given by

$$
u_{s}=\operatorname{sat}(u)=\left[\operatorname{sat}\left(u_{1}\right), \cdots, \operatorname{sat}\left(u_{m}\right)\right]^{T}
$$

where $\operatorname{sat}\left(u_{i}\right)=\operatorname{sign}\left(u_{i}\right) \times \min \left\{\left|u_{i}\right|, u_{\max , i}\right\}, u_{\max , i}>$ $0 \forall i \in\{i, \cdots, m\}$. Further, let $\mathcal{U}$ be given by:

$$
\mathcal{U}:=\mathcal{U}_{1} \times \cdots \times \mathcal{U}_{m}
$$

where $\mathcal{U}_{i}:=\left[-u_{\max , i}, u_{\max , i}\right]$. It is assumed that $u_{s}$ can be measures or estimated. To simplify the equations, it is assumed in the following, that the inputs has been scaled such that all inputs has the same saturation, i.e. $u_{\max , i}=u_{\max }$.

The saturation problem can be described by the general system setup given by (4). Let the input saturation be described by

$$
\begin{aligned}
u_{s}=\operatorname{sat}(u) & =\left(1+\psi_{\mathrm{sat}}(u)\right) u \\
\psi_{\text {sat }}(u) & =\operatorname{diag}\left(\psi_{1}\left(u_{1}\right), \cdots, \psi_{m}\left(u_{m}\right)\right)
\end{aligned}
$$

where $\psi_{i}\left(u_{i}\right) \in \mathcal{R}$ is a non-linear function given by:

$$
\psi_{i}\left(u_{i}\right)= \begin{cases}0 & \text { for } u_{i} \in \mathcal{U}_{i} \\ \frac{\operatorname{sat}\left(u_{i}\right)}{\left|u_{i}\right|}-1 & \text { for } u_{i} \notin \mathcal{U}_{i}\end{cases}
$$


Using this description of the saturation, it can be formulated in the general system set-up in (1). This is obtained by describing $u_{s}$ as:

$$
u_{s}=u+w_{s}, w_{s}=\psi_{\mathrm{sat}} z_{s}, z_{s}=u
$$

where $z_{s}$ is the input vector to $\psi_{\text {sat }}$ and output $w_{s}$ is the output vector from $\psi_{\text {sat }}$. The general system set-up in (13) with the above extensions is now given by:

$$
\Sigma_{S}:\left\{\left(\begin{array}{c}
z_{s} \\
\hline y \\
y_{s}
\end{array}\right)=\left(\begin{array}{c|c}
0 & I \\
\hline G_{y u} & G_{y u} \\
I & I
\end{array}\right)\left(\begin{array}{c}
w_{s} \\
u
\end{array}\right)\right.
$$

The feedback controller given by (5) is now given by:

$$
\Sigma_{C, S}:\left\{u=\left(\begin{array}{ll}
K & 0
\end{array}\right)\left(\begin{array}{c}
y \\
y_{s}
\end{array}\right)=K_{S}\left(\begin{array}{c}
y \\
y_{s}
\end{array}\right)\right.
$$

A coprime factorization of the above $\Sigma_{S}$ and $K_{S}$ are given by:

$$
\begin{aligned}
\left(\begin{array}{c}
G_{y u} \\
I
\end{array}\right) & =\left(\begin{array}{l}
N \\
M
\end{array}\right) M^{-1}=\left(\begin{array}{cc}
\tilde{M} & 0 \\
0 & I
\end{array}\right)^{-1}\left(\begin{array}{c}
\tilde{N} \\
I
\end{array}\right) \\
K_{S} & =\left(\begin{array}{ll}
U & 0
\end{array}\right)\left(\begin{array}{cc}
V & 0 \\
0 & I
\end{array}\right)^{-1}=\tilde{V}^{-1}(\tilde{U}
\end{aligned}
$$

by using (14).

With the coprime factorization given above, it is simple to give a complete description of the YJBK parameterized controller using the compact description given in Fig. 1. In this case, $\tilde{\mathcal{Z}}, \mathcal{Q}$ and $\varepsilon_{\mathcal{Q}}$ are given by:

$$
\begin{aligned}
\tilde{\mathcal{Z}} & =\left(\begin{array}{ccc}
\tilde{V}-I & -\tilde{U} & 0 \\
-\tilde{N} & \tilde{M} & 0 \\
-I & 0 & I
\end{array}\right) \\
\mathcal{Q} & =\left(\begin{array}{cc}
Q & Q_{s}
\end{array}\right) \\
\left(\begin{array}{l}
-u \\
\varepsilon_{\mathcal{Q}}
\end{array}\right) & =\left(\begin{array}{c}
-u \\
\varepsilon_{Q} \\
\varepsilon_{s}
\end{array}\right)=\tilde{\mathcal{Z}}\left(\begin{array}{c}
u \\
y \\
y_{s}
\end{array}\right)
\end{aligned}
$$

where $\varepsilon_{s}$ is the saturation error vector, i.e. $\varepsilon_{s}=u_{s}-u$ and $Q_{s}$ is the YJBK matrix transfer function for feedback control from the saturation error vector.

The formulation of input saturation as a special case of system extension gives a controller that can be implemented directly in the YJBK controller architecture shown in Fig. 1. Further, the anti-windup part of the controller is related only to the YJBK matrix transfer function $Q_{s}$ and separated from $Q$.

In the following, only the part of the parameterization related to the input saturation in the system is considered, i.e. $Q=0$. The case with $Q \neq 0$ is shortly discussed in Sec. $\mathrm{V}$.

\section{RELATION TO OTHER ANTI-WINDUP ARCHITECTURES}

The YJBK architecture including an anti-windup part is related to other anti-windup architectures. Here we will consider the architecture by Weston and Postlethwaite described in e.g. [4], [7], [13], [19] and the architecture by Kothare et.al. described in [6].
First, let's consider the W-P architecture. The setup is shown in Fig. 2, where $\Theta_{1}$ and $\Theta_{2}$ are the design parameters.

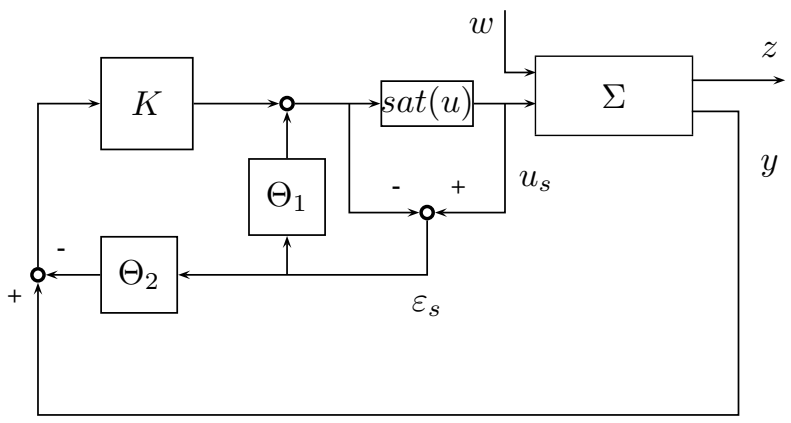

Fig. 2. The Weston-Postlethwaite controller architecture for systems with input saturation.

Note that the residual vector $\varepsilon_{s}$ given in (27), and in the W$\mathrm{P}$ controller architecture shown in Fig. 2 is the same vector. The control vector $u$ from the YJBK architecture in Fig. 1 with $\tilde{\mathcal{Z}}$ in (27) is given by:

$$
u=K y+\tilde{V}^{-1} Q_{s} \varepsilon_{s}
$$

The control input $u$ for the W-P architecture shown in Fig. 2 is given by:

$$
u=K y-K \Theta_{2} \varepsilon_{s}+\Theta_{1} \varepsilon_{s}
$$

Let (29) be rewritten into:

$$
u=K y-\tilde{V}^{-1}\left(\tilde{U} \Theta_{2}-\tilde{V} \Theta_{1}\right) \varepsilon_{s}
$$

It is now easy to see that the W-P anti-windup controller architecture described by (29) can be implemented in the YJBK architecture. Selecting $Q_{s}$ as:

$$
Q_{s}=-\tilde{U} \Theta_{2}+\tilde{V} \Theta_{1}=\left(\begin{array}{cc}
\tilde{V} & -\tilde{U}
\end{array}\right)\left(\begin{array}{c}
\Theta_{1} \\
\Theta_{2}
\end{array}\right)
$$

shows directly that two control vectors in (28) and (30) are identical. This shows that the W-P anti-windup controller can easily be implemented in the YJBK architecture. From [19], we have the following design of $\Theta_{1}$ and $\Theta_{2}$ are given by:

$$
\left(\begin{array}{c}
\Theta_{1} \\
\Theta_{2}
\end{array}\right)=\left(\begin{array}{l}
M-I \\
G_{y u} M
\end{array}\right)=\left(\begin{array}{c}
M-I \\
N
\end{array}\right)
$$

Based on these matrices, $Q_{s}$ take then the following form:

$$
\begin{aligned}
Q_{s} & =\left(\begin{array}{ll}
\tilde{V} & -\tilde{U}
\end{array}\right)\left(\begin{array}{c}
M-I \\
N
\end{array}\right) \\
& =\tilde{V} M-\tilde{V}-\tilde{U} N \\
& =I-\tilde{V}
\end{aligned}
$$

One of the important issues in the W-P architecture, compared with the YJBK architecture, is the implementation of the anti-windup part. An advantage in the W-P implementation, compared with the YJBK implementation, is that the additional control vectors from the anti-windup part are included in the loop before and after the controller. In the YJBK implementation, a single control input vector for the anti-windup part is injected into the nominal controller. This will in some cases not be acceptable because the nominal controller is modified.

It is therefore also relevant to consider the other way around. The starting point for calculating $\Theta_{1}$ and $\Theta_{2}$ based on $Q_{s}$ 
from the YJBK implementation is to consider (28). Using the Bezout equation in (7), $\tilde{V}^{-1}$ can be

$$
\tilde{V}^{-1}=M-\tilde{V}^{-1} \tilde{U} N=M-K N
$$

Using (34) in (28) gives directly:

$$
\begin{aligned}
u & =K y+(M-K N) Q_{s} \varepsilon_{s} \\
& =K y+M Q_{s} \varepsilon_{s}-K N Q_{s} \varepsilon_{s}
\end{aligned}
$$

Compare (35) with (29) gives directly that $\Theta_{1}$ and $\Theta_{2}$ are given by:

$$
\left(\begin{array}{c}
\Theta_{1} \\
\Theta_{2}
\end{array}\right)=\left(\begin{array}{c}
M \\
N
\end{array}\right) Q_{s}+\left(\begin{array}{c}
\Theta_{10} \\
\Theta_{20}
\end{array}\right)
$$

where $\Theta_{10}$ and $\Theta_{20}$ are free parameters that need to satisfy:

$$
\left(\begin{array}{ll}
\tilde{V} & -\tilde{U}
\end{array}\right)\left(\begin{array}{c}
\Theta_{10} \\
\Theta_{20}
\end{array}\right)=0
$$

Note that $\Theta_{1}$ and $\Theta_{2}$ are not unique when the calculation is based on $Q_{s}$ from the YJBK parameterization.

Note that the reformulation of the control input vector from the YJBK controller is not only restricted to the anti-windup case, but can also be used in the YJBK architecture.

The W-P anti-windup controller given by $Q_{s}$ in (33) has an interesting implementation in the general YJBK architecture. To see this, first, let the control vector in (28) be rewritten into:

$$
\tilde{V} u=\tilde{U} y+Q_{s} \varepsilon_{s}
$$

which gives

$$
u=\tilde{U} y-(\tilde{V}-I) u+Q_{s} \varepsilon_{s}
$$

(33) together with (37) gives now:

$$
\begin{aligned}
u & =\tilde{U} y-(\tilde{V}-I) u+(I-\tilde{V}) \varepsilon_{s} \\
& =\tilde{U} y-(\tilde{V}-I)\left(u+\varepsilon_{s}\right) \\
& =\tilde{U} y-(\tilde{V}-I) u_{s}
\end{aligned}
$$

by using $\varepsilon_{s}=u_{s}-u$.

Based on this control vector, the controller architecture from Fig. 1 takes the form as shown in Fig. 3.

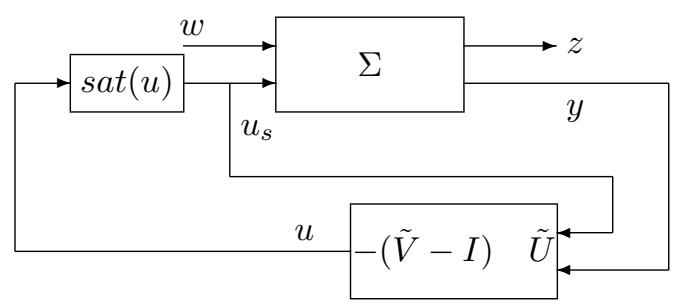

Fig. 3. The implementation of a feedback controller with anti-windup based on the left factored form.

The controller architecture showed in Fig. 3 is the same antiwindup architecture shown in [6]. The implementation showed in Fig. 3 demonstrates a simple way to include anti-windup in an existing controller. However, the controller still needs to be factorized and implemented using the coprime factors, so there is an internal feedback of the control vector. It is then simple to include the anti-windup part by moving the control feedback loop from the input side of the saturation block to the output side of the saturation block. The design freedom in this controller architecture is the coprime factorization of the controller. However, some controllers are implemented directly with this structure. An example of a controller with this structure is an observer based controller. The matrix transfer functions for the controller implementation shown in Fig. 3 are given by, [16]:

$$
\left(\begin{array}{cc|c}
-(\tilde{V}-I) & \tilde{U}
\end{array}\right)=\left(\begin{array}{c|cc}
A+L C_{y} & -\left(B_{u}+L D_{y u}\right) & L \\
\hline F & 0 & 0
\end{array}\right)
$$

where $F$ and $L$ are the state feedback gain and observer gain, respectively. An implementation of the full-order observer based feedback controller including an anti-windup part is shown in Fig. 4.

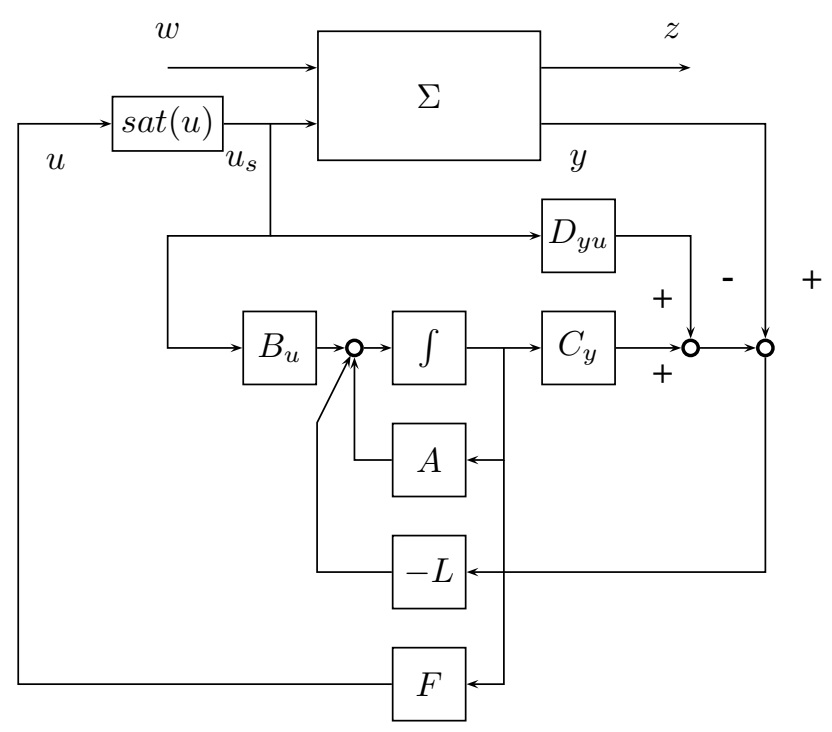

Fig. 4. An implementation on a full-order observer based feedback controller including an anti-windup part.

Fig 4 shows that the anti-windup controller from [6], or the W-P anti-windup controller given by the parameters in (32), can be implemented directly in an observer-based controller without including extra terms.

Using an observer-based feedback controller, it is possible to give a coprime factorization of system and controller as used in (39). A state space description for coprime factorization of general feedback controllers can be found in e.g. [16].

\section{Closed-Loop Analysis}

The stability analysis is based on the dual YJBK matrix transfer function $S(\Delta)$ given in (18). The closed-loop system is stable if the nominal closed-loop system is stable, and the dual YJBK matrix transfer function $S$ is stable, [9], [16]. Using $\Delta=\psi_{\text {sat }}$ where $\psi_{\text {sat }}$ is the non-linear function in (22) describing the input saturation. $S\left(\psi_{\text {sat }}\right)$ can be then calculated by using the system $\Sigma_{S}$ in (24), and the associated coprime matrices for the system in (26). $S\left(\psi_{\text {sat }}\right)$ is given by:

$$
S\left(\psi_{\text {sat }}\right)=\left(\begin{array}{c}
\tilde{N} \\
I
\end{array}\right) \psi_{\text {sat }}\left(I-U \tilde{N} \psi_{\text {sat }}\right)^{-1} M
$$


$S\left(\psi_{\text {sat }}\right)$ is stable if and only if

$$
\left(I-U \tilde{N} \psi_{\text {sat }}\right)^{-1} \in \mathcal{R} \mathcal{H}_{\infty}
$$

The stability can be checked using an LMI formulation, see e.g. [3].

Now, include $Q_{s}$ in the controller as described in (28). This gives the closed-loop:

$$
\begin{aligned}
S\left(Q_{s}, \psi_{\text {sat }}\right) & =S\left(\psi_{\text {sat }}\right)\left(I-\mathcal{Q} S\left(\psi_{\text {sat }}\right)\right)^{-1} \\
& =\left(\begin{array}{c}
\tilde{N} \\
I
\end{array}\right) \psi_{\text {sat }}\left(I-\left(U \tilde{N}+M Q_{s}\right) \psi_{\text {sat }}\right)^{-1} M
\end{aligned}
$$

The closed-loop is stable if:

$$
\left(I-\left(U \tilde{N}+M Q_{s}\right) \psi_{\text {sat }}\right)^{-1} \in \mathcal{R H}_{\infty}
$$

Consider the W-P controller given by $Q_{s}$ in (33). This gives the following stability condition:

$$
\left(I-(M-I) \psi_{\text {sat }}\right)^{-1} \in \mathcal{R H}_{\infty}
$$

This gives the same stability condition as given in [13], [18], [19]. Due to the fact that the coprime factorization is not unique, the coprime factorization of $G_{y u}$ needs to be optimized with respect to the anti-windup controller as described in [13], [18], [19].

Consider the stability condition in (43). This condition can be satisfied by using a $Q_{s}$ given by:

$$
Q_{s}=-M^{-1} U \tilde{N}=-\tilde{U} G_{y u}
$$

This $Q_{s}$ can only be applied if the system is open-loop stable. Further, it will decouple the controller and the saturation with respect to the closed-loop stability. This can be seen from (28) by including $Q_{s}$ from (45). The control vector is then given by:

$$
\begin{aligned}
u & =K y-\tilde{V}^{-1} \tilde{U} G_{y u} \varepsilon_{s} \\
& =K G_{y u} u_{s}-K G_{y u}\left(u_{s}-u\right) \\
& =K G_{y u} u
\end{aligned}
$$

This shows that the closed-loop system is stable if the system without saturation is closed-loop stable.

Until now, it has been assumed that the original YJBK matrix transfer function $Q$ in (27) is set to zero. Using a feedback controller based on the YJBK architecture, it is natural to apply $Q$ for optimization of closed-loop performance or robust stability. It is therefore relevant to consider the stability of the saturation loop for a non-zero $Q$. Including $\mathcal{Q}$ in the controller gives the following $S\left(\mathcal{Q}, \psi_{\text {sat }}\right)$ :

$$
S\left(\mathcal{Q}, \psi_{\text {sat }}\right)=\left(\begin{array}{c}
\tilde{N} \\
I
\end{array}\right) \psi_{\text {sat }}\left(I-\left(U \tilde{N}+M Q \tilde{N}+M Q_{s}\right) \psi_{\text {sat }}\right)^{-1}
$$

The stability of the saturation loop depends on $Q$. The extra term that occurs in (47) from a non-zero $Q$ can be compensated by selecting $Q_{s}$ as:

$$
Q_{s}(Q)=Q_{s}-Q \tilde{N}
$$

The influence from $Q$ on the stability of the saturation loop has then been removed completely. A redesign of the controller, using the YJBK matrix transfer function $Q$, can be done without a redesign of the anti-windup part of the controller.

\section{EXAMPLE}

Consider the system and controllers from [17] given by:

$$
\begin{aligned}
G_{y u, 0} & =\frac{10}{s^{2}+10 s+10} \quad G_{y u}=\frac{10}{s^{2}-0.05 s+10} \\
K_{\text {feedback }} & =\frac{135(s+5)^{2}}{s(s+80)} \quad K_{\text {forward }}=\frac{2.5}{s+2.5} K_{\text {feedback }}
\end{aligned}
$$

The maximal control input signal is limited to $u_{\max }=1.0$. $G_{y u, 0}$ is the nominal model used for design of the anti-windup controller where $G_{y u}$ is the system used in the simulations.

A simulation of the system with the nominal controller with and without saturation is shown in Fig. 5. The amplitude of the reference input is 1.1. The feedback system without saturation is closed loop stable whereas it is unstable when input saturation is included.

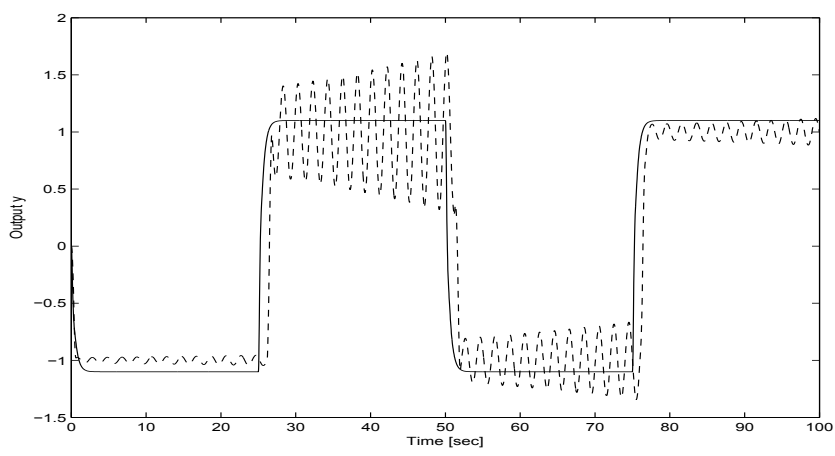

Fig. 5. Simulation of the system with the nominal controller. Solid line is the system without saturation and dashed line is system with saturation.

Let's consider three different anti-windup controllers for the system. The anti-windup parts are given by:

$$
Q_{s}= \begin{cases}I-\tilde{V} & \text { W-P controller } \\ k & \text { constant } Q_{s} \\ -G_{y u, 0} \tilde{U} & \text { decoupling controller }\end{cases}
$$

The constant $Q_{s}=k$ is designed by using a simple optimization. This gives $Q_{s}=10$. The simulation results of the three anti-windup controllers are shown in Fig. 6.
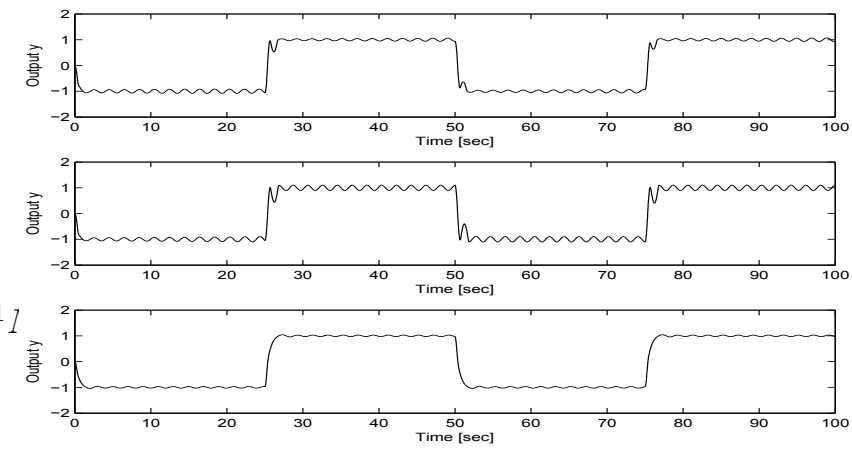

Fig. 6. Simulation of the system with three different anti-windup controllers. The top plot shows the output from the system $y$ when the W-P controller is applied, the middle plot shows $y$ when $Q_{s}=k$ is applied and the lower plot shows $y$ when the decoupling $Q_{s}$ is applied.

All three anti-windup controllers results in stable closedloop systems. The first two anti-windup controllers results in almost the same output with the first one as a little bit better 
than the second controller. The decoupling controller gives an output with a smaller oscillation than the other two controllers. The control signal generated by the anti-windup controllers are shown in Fig. 7. It can be seen from Fig. 7 that the W-P
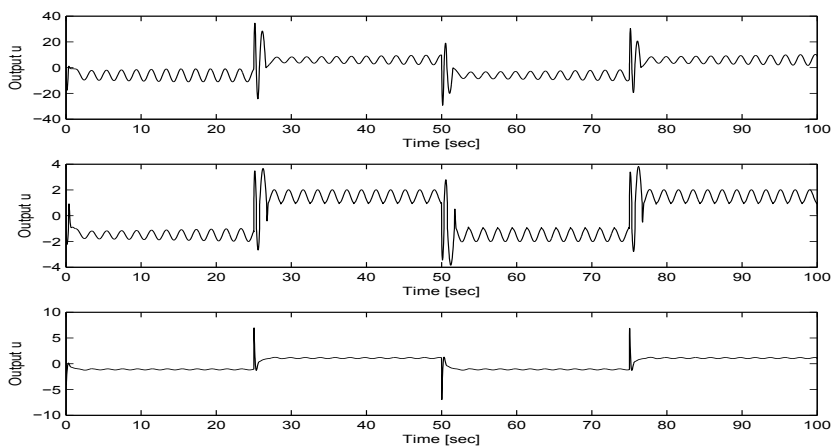

Fig. 7. Control signal from the three different anti-windup controllers. The top plot shows the control signal $u$ when the W-P controller is applied, the middle plot shows $u$ when $Q_{s}=k$ is applied and the lower plot shows $u$ when the decoupling $Q_{s}$ is applied.

controller results in a high control signal compared with the two other controllers. $Q_{s}=k$ results in reasonable control signal, and the decoupling controller gives a smaller and a more smooth signal control signal than from the two other controllers, but have some spikes.

It is important to point out that the above results depend strongly on the applied coprime factorization of the system and the nominal controller. Other coprime factorizations of the system and nominal controller will give other results, special for the first two anti-windup controllers.

\section{CONCLUSION}

A YJBK controller architecture has been applied in connection with input saturation. The YJBK architecture gives an additional YJBK transfer function related to the input saturation part of the system. This makes it easy to include anti-windup in the controller architecture. Standard analysis and design methods for input saturation can be applied.

Relations between the described YJBK architecture and other anti-windup controllers have also been considered. It is shown how the well-known Weston-Postlethwaite architecture can be implemented in the YJBK architecture, as well as the other way around.

\section{REFERENCES}

[1] J.M. Biannic and S. Tarbouriech. Optimization and implementation of dynamic anti-windup compensators with multiple saturation in flight control systems. Control Engineering Practice, 17:703-713, 2009.

[2] S. Galeani, S. Tarbouriech, M. Turner, and L. Zaccarian. A tutorial on modern anti-windup design. Euopean Journal of Control, 15(3-4):418440,2009

[3] G. Grimm, J. Hatfield, I. Postlethwaite, A.R. Teel, and L. Zaccarian. Anti-windup for stable linear systems with input saturation: An LMI based solution. IEEE Transactions on Automatic Control, 48(9):1509$1525,2003$.

[4] G. Herrmann, M.C. Turner, and I. Postlethwaite. Discrete-time and sampled-data anti-windup synthesis: stability and performance. Int. J. od Systems Science, 37(2):91-113, 2006.

[5] T. Hu, A.R. Teel, and L. Zaccarian. Anti-windup synthesis for linear control systems with input saturation: Achieving regional, nonlinear performance. Automatica, 44:512-519, 2008.
[6] M.V. Kothare, M. Morari, and C.N. Nett. A unified framework for the study of anti-windup designs. Automatica, 30(12):1869-1883, 1994.

[7] A. Marcos, M.C. Turner, and I. Postlethwaite. An architecture for design and analysis of high-performance robust antiwindup compensators. IEEE Transactions on Automatic Control, 52(9):1672-1679, 2007.

[8] E. Mulder, M. Kothare, and M. Morari. Multivariable anti-windup controller synthesis using linear matrix inequalities. Automatica, 37:14071416, 2001.

[9] H.H. Niemann. Dual Youla parameterization. IEE Proceedings - Control Theory and Applications, 150(5):493-497, 2003.

[10] H.H. Niemann. Parameterization of extended systems. IEE Proceedings - Control Theory and Applications, 153(2):221-227, 2006.

[11] H.H. Niemann. An architecture for controller parameterization. In Proceedings of the American Control Conference, pages 418-423, Milwaukee, WI, USA, 2018.

[12] S. Skogestad and I. Postlethwaite. Multivariable feedback control: Analysis and Design. Wiley, 2005.

[13] J. Sofrony, M.C. Turner, and I. Postlethwaite. Anti-windup synthesis using Riccati equation. International Journal of Control, 80(1):112128, 2007.

[14] K. Tabaka. Analysis and synthesis of anti-windup control system based on Youla parameterization. In Proceedings of the 41st IEEE Conference on Decision and Control, pages 2692-2697, Las Vegas, NV, USA, 2002.

[15] S. Tarbouriech and M.C. Turner. Anti-windup design: an overview of some recent advances and open problems. IET Control Theory and Applications, 3(1):1-19, 2009.

[16] T.T. Tay, I.M.Y. Mareels, and J.B. Moore. High performance control. Birkhäuser, 1997.

[17] M.C. Turner, G. Herrmann, and I. Postlethwaite. Incorporating robustness requirements into antiwindup design. IEEE Transactions on Automatic Control, 52(10):1842-1855, 2007.

[18] M.C. Turner and I. Postlethwaite. A new perspective on static and low order anti-windup synthesis. International Journal of Control, 77(1):2744, 2004.

[19] P.F. Weston and I. Postlethwaite. Linear conditioning for systems containing saturating actuators. Automatica, 36:1347-1354, 2000.

[20] L. Zhong-Shen, M. Xiao-Qin, G. Shang-Jia, L. Wei-Yao, and H. ChunQing. 4-degree-of-freedom anti-windup scheme for plants with actuatorsaturation. Journal of Process Control, 47:111-120, 2016. 